\title{
Nuevas combinaciones, una epitipificación y una corrección nomenclatural en Thelocephala (Cactoideae: Notocacteae): género endémico de Chile
}

\author{
New combinations, an epityfication and a nomenclatural correction in Thelocephala \\ (Cactoideae: Notocacteae): an endemic genus of Chile
}

\author{
Patricio Saldivia Pérez \& Luis Faúndez Yancas
}

Laboratorio de Morfología y Taxonomía Vegetal, Facultad de Ciencias Agronómicas, Universidad de Chile. Av. Santa Rosa 11315, Casilla 1004, La Pintana, Santiago, Chile.

BIOTA, Gestión y Consultorías Ambientales Limitada, Roberto Owen 0267, La Florida, Santiago, Chile.

patricio.saldivia.perez@gmail.com

\begin{abstract}
Within the revision works of the Thelocephala genus it is necessary to carry out new combinations for generic entities recently described under the broad concept of Eriosyce. Additionally, it has been made a correction for one of the binomies used by F. Ritter in 1980 and an epitype is designated for one of the recent taxa.
\end{abstract}

Entre las cactáceas chilenas, aquéllas globulares de la tribu Notocacteae (sensu Nyffeler \& Eggli 2010), han sido motivo de diversos arreglos sistemáticos a nivel genérico con una historia taxonómica, en el mejor de los casos, controvertida.

Así, los géneros Pyrrhocactus A.Berger sensu Ritter y Neoporteria Britton \& Rose, Islaya Backeb. y Thelocephala Y.Itô en el sentido estricto, han sido agrupados en Neoporteria (Donald \& Rowley 1966) y posteriormente reunidos con Eriosyce (Kattermann 1994), manteniéndose este último nombre en razón de su prioridad. En consideración de que dichas propuestas han planteado agrupamientos muy amplios (forzados) de morfología y ecología diversa que dan cuenta de linajes independientes y de que no colaboran a un entendimiento taxonómico ni biológico del grupo, en el reciente catálogo de Cactaceae para la flora del Cono Sur (Kiesling et al. 2008) se han considerado los géneros mencionados en forma independiente.

Dentro de éstos, Thelocephala Y.Itô corresponde al grupo que presenta mayores problemas respecto a los riesgos a su estado de conservación, particularmente por lo restringido de su distribución y por las presiones ambientales actuales, por lo cual su ordenamiento taxonómico resulta fundamental para establecer las entidades biológicas que lo integran.

Thelocephala fue creado por Yoshi Itô en 1957, y posteriormente enmendado bajo el nombre genérico Chileorebutia (Ritter 1959) y revisado rigurosamente por Friedrich Ritter en su extensa monografía de las cactáceas chilenas (Ritter 1980), en donde reconoce 15 especies y una probable nueva entidad específica. El género incluye plantas caracterizadas por su hábito geófito de raíz napiforme con o sin adelgazamiento del cuello, con el cuerpo generalmente sin costillas, cubierto por tubérculos dispuestos en líneas helicoidales y con la porción superior plana en la que se encuentran dispuestas las areolas, normalmente hundidas. Espinas radiales (raro una central) reducidas hasta ausentes en la madurez, reflejas lateralmente. Flores infundibuliformes con diámetros similares a los tallos, a veces algo mayores a ellos. Axilas de las escamas del pericarpelo y fruto con densos copos de lana y frecuentemente filamentos pilosos. El fruto representa una de las características evolutivas de mayor interés, ya que se encuentra adaptado para una dispersión anemócora, diseminando las semillas a medida que éste es arrastrado por el viento, no así en Pyrrhocactus, el género más emparentado, en el cual las semillas se desprenden inmediatamente con la liberación del fruto al sustrato y/o generalmente se apilan sobre el ápice de la planta madre. El género es endémico del desierto costero y llanos interiores hasta los 600-700 msnm aproximadamente, desde el sur de la Región de Antofagasta hasta el norte de la de Coquimbo.

En el marco de la revisión taxonómica de Thelocephala, se han reconocido algunos taxa recientes de Eriosyce (sensu lato) como representantes claros del género en estudio a nivel específico, por lo que se hace necesario realizar las combinaciones correspondientes. Adicionalmente, se realiza una corrección nomenclatural a uno de los nombres establecidos por Ritter (1980) para el género y se designa un epitipo para uno de los taxa de descripción reciente. 
NUEVAS COMBINACIONES Y EPITIPIFICACIÓN

Thelocephala weisseri (A.E.Hoffm. \& Helmut Walter) Faúndez \& Saldivia comb. et stat. nov. Basónimo: Eriosyce odieri (Lem. ex Salm-Dyck) Katt. subsp. malleolata (F.Ritter) A.E.Hoffm. \& Helmut Walter var. weisseri A.E.Hoffm. \& Helmut Walter, Gayana Bot. 63(1): 124. 2006. TIPO: Chile, Prov. Antofagasta, Cifuncho. 1987. Adriana Hoffmann s/n (holotipo SGO 152412!). - Epitipo (aquí designado): Chile, Región de Antofagasta, Prov. Antofagasta, camino Cifuncho - Pta. Tórtolas, 503 m. 13-X-2010. L. Faúndez s/n \& P. Saldivia 923 (SGO 160437!).

El material tipo depositado en SGO corresponde únicamente a un fruto inmaduro seco en donde sólo se puede apreciar el indumento del pericarpelo acompañado de una foto de un ejemplar vegetativo en hábitat. En consideración de que dicho material no permite un adecuado trabajo ni clarificación de los caracteres diagnósticos de la especie (Art. 9.7; McNeill et al. 2006), se optó por designar como epitipo un nuevo material que consta de una planta completa en flor.

Thelocephala challensis (I.Schaub \& Keim) Faúndez \& Saldivia comb. et stat. nov. Basónimo: Eriosyce napina (Phil.) Katt. subsp. challensis I.Schaub \& Keim, Cactus \& Co. 9(2): 111. 2005. TIPO: Chile, III Región, South east of Carrizal Bajo, 165 a 290 msnm, 20-VIII-2004, I. Schaub \& R. Keim (holotipo SGO 151590!).

Material ADicional

CHILE, Región de Atacama, Prov. Huasco, Parque Nacional Llanos de Challe, 70 m, 11- X-2010, L. Faúndez s/n \& P. Saldivia 898 (SGO) 160436.

En la descripción original, Schaub \& Keim (2005) señalan erróneamente a la localidad tipo dentro de la Región de Coquimbo.

Thelocephala riparia (Mächler \& Helmut Walter) Faúndez \& Saldivia comb. et stat. nov. Basónimo: Eriosyce napina (Phil.) Katt. subsp. riparia Mächler \& Helmut Walter, Cact. World 24(3): 142. 2006. TIPO: Chile, Prov. Elqui, east of Trapiche, 3-XI-2004, Helmut Walter 487 (holotipo SGO 152410 !).

Thelocephala monte-amarguensis (Katt.) Faúndez \& Saldivia comb. et stat. nov. Basónimo: Eriosyce odieri (Lem. ex Salm-Dyck) Katt. var. monte-amargensis Katt., Eriosyce (Cactac.) gen. revis. \& ampl. (Succ. Pl. Res., 1) 118. 1994. TIPO: Chile, Copiapó, S of Monte Amargo, FK - 522 (holotipo DBG no visto).

MATERIAL ADICIONAL

CHILE, Región de Atacama, Prov. Copiapó, Monte Amargo, $27^{\circ} 21^{\prime} \mathrm{S}-70^{\circ} 44^{\prime} \mathrm{W}, 137 \mathrm{~m}$, sin fecha, Ritter 503 loc. 1 (SGO) 125134.

\section{CORRECCIÓN NOMENCLATURAL}

Thelocephala lembckei (Katt.) Faúndez \& Saldivia comb. et stat. nov. Basónimo: Eriosyce napina (Phil.) Katt. subsp. lembckei Katt. var. lembckei Katt., Eriosyce (Cactac.) (Succ. Pl. Res. 1): 118. 1994. TIPO: Chile. Huasco, W of Freirina, FK - 77 (holotipo DBG no visto).

\section{Material ADICIONAL}

CHILE, Región de Atacama, Prov. Huasco: Oeste de Vallenar, 28 34'S-7047'W, 373 m, sin fecha, Ritter 501 loc. 3 (SGO) 125136; Prov. Huasco: Quebrada Maitencillo, planicies sur, $261 \mathrm{~m}, 11-\mathrm{X}-2010$, L. Faúndez s/n \& P. Saldivia 897 (SGO) 160438.

Thelocephala lembckei es un nombre aplicado inválidamente por Ritter (T. lembckei (Backeb.) F. Ritter (Ritter, Kakteen Südamerika 3: 1005. 1980)), ya que se basa en Neochilenia lembckei Backeb. (Backeberg, Cactaceae 3: 1822. 1959); nombre no válidamente publicado por no designar material tipo (Art. 37.1; McNeill et al. 2006). Luego, Kattermann (1994) es quien valida la entidad dentro de Eriosyce (sensu lato) a nivel infraespecífico al citar holotipo. Se ha optado por mantener el binomio empleado por Ritter, ya que corresponde al epíteto específico ampliamente utilizado para su denominación.

\section{AGRADECIMIENTOS}

Al Dr. Roberto Kiesling por su apoyo y orientación permanentes, además del envío de literatura fundamental. A las Sras. Gloria Rojas y Mélica Muñoz por las facilidades prestadas en la consulta de materiales en SGO. A Tomasz Romulski por el envío de literatura y a Jimena Martínez por la traducción del resumen. Así mismo, a los revisores anónimos, cuyos aportes ayudaron a mejorar la redacción y comprensión de los cambios propuestos.

\section{BIBLIOGRAFÍA}

BACKeberg, C. 1959. Die Cactaceae: Handbuch der Kakteenkunde. Band 3. Cereoideae (Austrocactinae). Veb Gustav Fischer Verlag. Jena. (xxv-xxx), 1361-1926, (1-4), lám. 108-160.

Donald, J.D. \& G.D. Rowley. 1966. Reunion of the genus Neoporteria. Cactus and Succulent Journal of Great Britain 28(3-4): 54-63.

ITo, Y. 1957. Explanatory Diagram of Austroechinocactinae. Japan Cactus Laboratory. Japan. 319 pp.

Kattermann, F. 1994. Eriosyce (Cactaceae). The genus revised and amplified. Succulent Plant Research 1: 1-176.

Kiesling, R., J. Larroca, L. Faúndez, D. Metzing \& S. Albesiano. 2008. Cactaceae. En: F.O. Zuloaga, O. Morrone \& M.J. Belgrano (eds.), Catálogo de las Plantas Vasculares del Cono Sur de América (Argentina, Sur de Brasil, Chile, Paraguay y Uruguay). Volumen 2. Dicotyledonae: 
Acanthaceae - Fabaceae (Abarema - Schizolobium). Monographs in Systematic Botany from the Missouri Botanical Garden 107: 1715-1830.

McNeill, J., F.R. Barrie, H.M. Burdet, V. Demoulin, D.L. Hawksworth, K. Marhold, D.H. Nicolson, J. Prado, P.C. Silva, J.E. Skog, J.H. Wiersema \& N.J. Turland. 2006. International Code of Botanical Nomenclature (Viena Code). Regnum Vegetabile 146. A.R.G. Gantner, Verlag KG. VIII + 568 pp.

Nyffeler, R. \& U. EgGLi. 2010. A Farewell to dated ideas and concepts: molecular phylogenetics and a revised suprageneric classification of the family Cactaceae. Schumannia 6: 109-149.
Ritter, F. 1959. Chileorebutia Ritter gen. nov. ex Fric pro parte. Cactus, Organe de l'Association Francaise de Cactus et Plantes Grasses, Paris. 65: 191-194.

Ritter, F. 1980. Kakteen in Südamerika. Band 3. Chile. Spangenberg. Pp. 857-1238.

Schaub, I. \& R. Keim. 2005. A new 'thelocephala' Eriosyce napina ssp. challensis. Cactus \& Co. 9 (2): 104-114.

WALter, H. \& W. Mächler. 2006. Rearrangements in the systematic of Eriosyce napina (Philippi) Kattermann (Cactaceae). CactusWorld 24 (3): 135-143.

WAtson, J.M. 2006. Validation of some recent Chilean taxonomic novelties in Eriosyce Phil. (Cactaceae). Gayana Botánica 63(1): 123-125.

Recibido: 19.04.11

Aceptado: 15.06.11 